\title{
Gut microbiota and neoplastic diseases of the gastrointestinal tract
}

\author{
Aleksandra Pilśniak', Urszula Dworzecka', Ewa Otto-Buczkowska² \\ 'Maria Sklodowska-Curie National Research Institute of Oncology, Gliwice Branch, Poland \\ ${ }^{2}$ Medical Specialist Centre in Gliwice, Poland
}

The term "microbiome" is used to describe the substantial number and diverse spectrum of microorganisms that inhabit the body. It plays an essential role in health conditions and diseases. Recent years have brought a further intensification of experimental studies on the impact of the microbiome on the human body, particularly with the aim of identifying and clarifying this impact. Many studies indicate that diet, lifestyle and drugs can affect the composition of the intestinal microflora, which, in turn, can modulate the development and progression of gastrointestinal tract tumors. It is suspected that the gut microbiome plays a significant role in the formation of gastrointestinal tumors. On the other hand, the role of the intestinal microflora in inhibiting the processes of oncogenesis suggests that this mechanism may be used to prevent and treat gastrointestinal cancer. Using probiotics to modify the microbiome may be beneficial in cancer therapy and may be used as a supportive treatment for classic cancer therapies such as chemotherapy, radiotherapy and surgical treatment. Intestinal microbiome analysis can be potentially used to develop non-invasive diagnostic tests. These tests could be useful as new protective markers for colorectal cancer, or as prognostic markers and predictive markers of response to treatment, especially immunotherapy.

Key words: microbiome, gastrointestinal cancer, probiotics

\section{Introduction}

The term "microbiome" is used to describe the substantial number and diverse spectrum of microorganisms that inhabit the body. It was suggested by Joshua Lederberg in 2001 to cover the entire population of commensal, symbiotic and pathogenic microorganisms. It has been established that with a mass of about $2 \mathrm{~kg}$, the number of cells in the microbiome exceeds the number of cells that make up the healthy human body by 10 times. It plays an essential role in health conditions and diseases. Recent years have brought a further intensification of experimental studies on the impact of the microbiome on the human body, particularly with the aim to identify and clarify this impact.

Each part of the body is inhabited by a specific microbial population. The biggest and most heterogenous is that of the gut microbiota. This plays an important role in many diseases of the digestive and other systems, and exhibits a broad spectrum of actions, including effects on the immune system. Toll-like receptors (TLR) are sensors of infections caused by microorganisms and the microbiome, which play a major role in identifying the threat and initiating the inflammation and immune response. The intestinal microbiome stimulates both specific and non-specific immune mechanisms of the body. It is also involved in regulating immune responses. It affects the lymphoid tissue associated with the mucosal associated lymphoid tissue (MALT) and stimulates the synthesis of antibodies. Saprophytic bacteria of the gastrointestinal tract inhibit inflammation and affect the tightening of the intestinal epithelial barrier [1, 2].

It has been found that gastrointestinal cancers account for up to one third of all cancer diseases. A number of factors are

\section{How to cite:}

Pilśniak A, Dworzecka U, Otto-Buczkowska E. Gut microbiota and neoplastic diseases of the gastrointestinal tract. NOWOTWORY J Oncol $2020 ; 70: 150-152$. 
involved in oncogenesis - both extracellular, and intracellular, such as cellular membrane proteins and transmembrane proteins localized in the cytosol or nucleus. Many studies indicate that diet, lifestyle and drugs can affect the composition of the intestinal microflora, which, in turn, can modulate the development and progression of gastrointestinal tract tumors. Therefore, it is suspected that the gut microbiome plays a significant role in the formation of gastrointestinal tumors [3-5].

Intestinal bacteria stimulate the production of the tumor necrosis factor (TNF), which affects the activity of lymphocytes, cell metabolism, as well as the apoptosis of cancer cells. They can enhance the development and progression of gastrointestinal tumors by damaging DNA, activating oncogenic signaling pathways, producing tumor-stimulating metabolites such as secondary bile acids, and suppressing anti-tumor immunity [6].

It has been found that metabolites, such as the secondary fatty acids produced by some bacterial species, may promote the development of gastrointestinal tumors [7]. Experimental studies have identified microorganisms that can promote oncogenesis by increasing cell proliferation and the production of metabolites such as butyrate [8]. In contrast, the short-chain fatty acids (SCFA) produced by other species play a suppressing role in cell proliferation and cell apoptosis induction processes and are responsible for maintaining balance in anti-inflammatory and pro-inflammatory reactions. They are characterized by the induction of T-regulatory cells (Treg) through free fatty acid receptors (GPR). To summarize, SCFA can suppress inflammatory processes and oncogenesis. In particular, a high concentration of butyric acid may inhibit oncogenesis. Cancer cells are characterized by a high rate of proliferation and the anti-tumor activity of butyric acid is based on inhibiting this proliferation.

Recently presented research results show that some bacterial species produce metabolites such as secondary bile acids, and an increased concentration of these intensifies the development of gastrointestinal tumors due to their cytotoxicity [7].

The role of the intestinal microflora in inhibiting the processes of oncogenesis suggests that this mechanism may be used in preventing and treating gastrointestinal cancer.

\section{The use of probiotics in preventing and treating cancerous diseases of the gastrointestinal tract}

There is a connection between the intestinal microflora, its metabolic activity and the mode of nutrition. Combined with genetic predisposition, unfavorable environmental factors and bad eating habits may disturb the composition of the gastrointestinal microflora. Therefore, research is being carried out on the use of probiotics to modify the disbalance of gut microbiota [7]. Negative metabolic changes induced by intestinal microorganisms can cause toxic oncogenic substances to form; these, in turn, may contribute to the development of cancer [9].
Administering probiotics increases the pool of beneficial intestinal microflora, and thus seems to create conditions for limiting changes in the intestines [10-12]. Researchers from Italy have presented a discussion of the results of studies which had been conducted on this problem [13]. Their review suggests that probiotics may reduce the risk of cancer through a number of mechanisms, including the degradation of potential carcinogenic factors and the production of anti-cancer compounds.

Another literature review presents the results of research conducted on the impact of probiotics on the suppression of gastrointestinal and other cancers, and also on their mechanisms of action [14]. Other authors have also presented a review of literature on the mechanisms of probiotics in neoplastic diseases of the gastrointestinal tract [15]. Though numerous studies conducted on animal models can serve as evidence to the beneficial effects of probiotics in the prevention of neoplastic processes, it is necessary to conduct extensive clinical trials on humans to determine potential bacterial strains, dosages and schedules of administration depending on the types and stages of cancer development $[16,17]$.

The fecal microbiota transplant (FMT), which is a method used to cure specific diseases by reconstructing normal functioning and the immune system, is also worth mentioning in this context. Its influence on the recipient's immune system is complicated and unpredictable, so further investigation is necessary to answer numerous questions which still remain [18].

Among the modern drugs used in cancer therapy, much attention has been given to immunological drugs, i.e. to anti-CTLA4, anti-PD-1 and anti-PD-L1 inhibitors, whose action is designed to stimulate the immune system [19]. The problem is that only those patients who are positive for the CTLA4 protein, the so-called programmed death receptor 1 (PD-1) and its ligand (PD-L1) are eligible for such treatment. However, the effectiveness of microflora participation in controlling these activities requires further research [20].

Many studies indicate that the intestinal microflora not only plays a role in the formation of cancer, but also modifies the effectiveness of therapy [19]. Recently, research has been presented on the regulation of the composition and methods of using probiotics in patients undergoing chemotherapy and radiotherapy [18]. Suggestions to use the microflora as supportive treatment for other cancer therapies, such as chemotherapy and immunotherapy, are also being considered $[16,17]$.

There are also studies that show the benefits of regulating the intestinal microflora through the use of probiotics in patients treated oncologically with 5-fluorouracil (5-FU), which is one of the chemotherapeutic agents used in the treatment of cancer. This drug significantly damages the microflora, and, therefore, its correction with the use of probiotics is highly desirable [21]. In addition to the unquestionable benefits of supra-cancer therapy, the use of probiotics may also cause adverse effects in immunocompromised patients. Attempts are being made to 
develop the possibility of individually selecting bacterial species, taking into account the specific needs of each patient. That kind of program would allow for obtaining the beneficial effects of using probiotics while avoiding side effects [22, 23].

Wieczorska et al. emphasize that intestinal microbiome analysis can be potentially used to develop non-invasive diagnostic tests. These tests could be useful as new protective markers for colorectal cancer, or as prognostic markers and predictive markers of the response to treatment, especially immunotherapy $[24,25]$.

\section{Conclusions}

At present, it is generally accepted that intestinal bacteria have an important influence on the cancer process. Depending on the composition of the microbiome, this influence may intensify cancer processes; however, it may also consist in protective functions, as well as preventing or slowing oncogenesis when the composition of the microbiome changes. The research conducted shows that modifying the microbiome using probiotics may be beneficial in cancer therapy and may be used as a supportive treatment for classic cancer therapies such as chemotherapy, radiotherapy and surgical treatment. Extensive clinical trials are nonetheless required to identify the dosages and administration regimes as a supportive or alternative cancer treatment. Further work is also needed on re-selecting systems for the selection of optimal microbiome compositions for the individual needs of each patient, i.e. on so-called microbiological biological engineering [26].

\section{Conflict of interest: none declared}

\section{Aleksandra Pilśniak}

Maria Sklodowska-Curie National Research Institute of Oncology Gliwice Branch

Wybrzeże Armii Krajowej 15

44-102 Gliwice, Poland

e-mail:dudek.ola@op.pl

\section{Received: 4 Apr 2020}

Accepted: 15 Apr 2020

\section{References}

1. Majewska M, Szczepanik M. [The role of Toll-like receptors (TLR) in innate and adaptive immune responses and their function in immune response regulation]. Postepy Hig Med Dosw (Online). 2006; 60: 52-63, indexed in Pubmed: 16474276.

2. Majewska M, Szczepanik M. [Contact sensitivity reaction, its mechanism and regulation]. Postepy Hig Med Dosw (Online). 2009; 63: 47-57, indexed in Pubmed: 19252464.

3. Sonnenburg ED, Smits SA, Tikhonov M, et al. Diet-induced extinctions in the gut microbiota compound over generations. Nature. 2016; 529(7585): 212-215, doi: 10.1038/nature16504, indexed in Pubmed: 26762459.

4. Zhernakova A, Kurilshikov A, Bonder MJ, et al. LifeLines cohort study. Population-based metagenomics analysis reveals markers for gut microbiome composition and diversity. Science. 2016; 352(6285): 565-569, doi: 10.1126/science.aad3369, indexed in Pubmed: 27126040.

5. Alhinai EA, Walton GE, Commane DM. The Role of the Gut Microbiota in Colorectal Cancer Causation. Int J Mol Sci. 2019; 20(21), doi: 10.3390/ ijms20215295, indexed in Pubmed: 31653078.
6. Mima K, Ogino S, Nakagawa S, et al. The role of intestinal bacteria in the development and progression of gastrointestinal tract neoplasms. Surg Oncol. 2017; 26(4): 368-376, doi: 10.1016/j.suronc.2017.07.011, indexed in Pubmed: 29113654.

7. O'Keefe SJD. Diet, microorganisms and their metabolites, and colon cancer. Nat Rev Gastroenterol Hepatol. 2016; 13(12): 691-706, doi: 10.1038/nrgastro.2016.165, indexed in Pubmed: 27848961.

8. Abreu MT, Peek RM. Gastrointestinal malignancy and the microbiome. Gastroenterology. 2014; 146(6): 1534-1546.e3, doi: 10.1053/j. gastro.2014.01.001, indexed in Pubmed: 24406471.

9. Louis P, Hold GL, Flint HJ. The gut microbiota, bacterial metabolites and colorectal cancer. Nat Rev Microbiol. 2014; 12(10): 661-672, doi: 10.1038/nrmicro3344, indexed in Pubmed: 25198138.

10. Dos Reis SA, da Conceição LL, Siqueira NP, et al. Review of the mechanisms of probiotic actions in the prevention of colorectal cancer. Nutr Res. 2017; 37: 1-19, doi: 10.1016/j.nutres.2016.11.009, indexed in Pubmed: 28215310.

11. Wasilewska E, Złotkowska D, Pijagin ME. [The role of intestinal microflora and probiotic bacteria in prophylactic and development of colorectal cancer]. Postepy Hig Med Dosw (Online). 2013; 67: 837-847, doi: 10.5604/17322693.1061847, indexed in Pubmed: 24018449.

12. $\mathrm{Yu} \mathrm{AQ,} \mathrm{Li} \mathrm{L.} \mathrm{The} \mathrm{Potential} \mathrm{Role} \mathrm{of} \mathrm{Probiotics} \mathrm{in} \mathrm{Cancer} \mathrm{Preven-}$ tion and Treatment. Nutr Cancer. 2016; 68(4): 535-544, doi: 10.1080/01635581.2016.1158300, indexed in Pubmed: 27144297.

13. Orlando A, Russo F. RETRACTED ARTICLE: Intestinal Microbiota, Probiotics and Human Gastrointestinal Cancers. J Gastrointest Cancer. 2012; 44(2): 121-131, doi: 10.1007/s12029-012-9459-1.

14. So SSY, Wan MLY, El-Nezami H. Probiotics-mediated suppression of cancer. Curr Opin Oncol. 2017; 29(1): 62-72, doi: 10.1097/ CCO.0000000000000342, indexed in Pubmed: 27792053.

15. Javanmard A, Ashtari S, Sabet B, et al. Probiotics and their role in gastrointestinal cancers prevention and treatment; an overview. Gastroenterol Hepatol Bed Bench. 2018; 11(4): 284-295, indexed in Pubmed: 30425806.

16. Gopalakrishnan V, Helmink BA, Spencer CN, et al. The Influence of the Gut Microbiome on Cancer, Immunity, and Cancer Immunotherapy. Cancer Cell. 2018; 33(4): 570-580, doi: 10.1016/j.ccell.2018.03.015, indexed in Pubmed: 29634945.

17. McQuade JL, Daniel CR, Helmink BA, et al. Modulating the microbiome to improve therapeutic response in cancer. Lancet Oncol. 2019; 20(2): e77-e91, doi: 10.1016/S1470-2045(18)30952-5, indexed in Pubmed: 30712808.

18. Ding C, Tang W, Fan X, et al. Intestinal microbiota: a novel perspective in colorectal cancer biotherapeutics. Onco Targets Ther. 2018; 11: 4797-4810, doi: 10.2147/OTT.S170626, indexed in Pubmed: 30147331.

19. Andrews MC, Wargo JA. Cancer Evolution during Immunotherapy. Cell. 2017; 171(4): 740-742, doi: 10.1016/j.cell.2017.10.027, indexed in Pubmed: 29100071.

20. Pouncey AL, Scott AJ, Alexander JL, et al. Gut microbiota, chemotherapy and the host: the influence of the gut microbiota on cancer treatment. Ecancermedicalscience. 2018; 12: 868, doi: 10.3332/ecancer.2018.868, indexed in Pubmed: 30263059.

21. Singh S, Kotla NG, Tomar S, et al. A nanomedicine-promising approach to provide an appropriate colon-targeted drug delivery system for 5-fluorouracil. Int J Nanomedicine. 2015; 10: 7175-7182, doi: 10.2147/ IJN.S89030, indexed in Pubmed: 26648721.

22. Vivarelli S, Salemi R, Candido S, et al. Gut Microbiota and Cancer: From Pathogenesis to Therapy. Cancers (Basel). 2019; 11(1), doi: 10.3390/ cancers11010038, indexed in Pubmed: 30609850.

23. Nagano T, Otoshi T, Hazama D, et al. Novel cancer therapy targeting microbiome. Onco Targets Ther. 2019; 12: 3619-3624, doi: 10.2147/ OTT.S207546, indexed in Pubmed: 31190864.

24. Wieczorska K, Stolarek M, Stec R. The Role of the Gut Microbiome in Colorectal Cancer: Where Are We? Where Are We Going? Clin Colorectal Cancer. 2020; 19(1): 5-12, doi: 10.1016/j.clcc.2019.07.006, indexed in Pubmed: 31678050.

25. Wong SH, Yu J. Gut microbiota in colorectal cancer: mechanisms of action and clinical applications. Nat Rev Gastroenterol Hepatol. 2019; 16(11):690-704, doi: 10.1038/s41575-019-0209-8, indexed in Pubmed: 31554963.

26. Meng C, Bai C, Brown T, et al. Human Gut Microbiota and Gastrointestinal Cancer. Genomics, Proteomics \& Bioinformatics. 2018; 16(1): 33-49, doi: 10.1016/j.gpb.2017.06.002. 